\title{
Noten für Kliniken im Internet abrufbereit Transparente Behandlungsqualität im Krankenhaus
}

Seit dem Jahr 2003 sind Krankenhäuser gesetzlich verpflichtet, regelmäßig strukturierte Qualitätsberichte zu veröffentlichen. Die Berichte sollen der Information von Patienten sowie von einweisenden Ärzten dienen. Krankenhäusern eröffnen sie die Möglichkeit, Leistungen und Qualität darzustellen und damit um das Vertrauen der Patienten zu werben.

Zuständig für die Veröffentlichung der Daten zur Behandlungsqualität an deutschen Krankenhäusern ist bis zu diesem Jahr die Bundesgeschäftsstelle Qualitätssicherung (BQS). Sie hat zuletzt Ende 2008 den Qualitätsreport für das Jahr 2007 veröffentlicht. Auf 208 Seiten werden in dem Bericht die interessantesten Ergebnisse für 100 der 194 Qualitätsindikatoren aus 26 Bereichen des Krankenhausgeschehens dargestellt. Sie basieren auf fast 3,6 Millionen Datensätzen von mehr als 1600 Krankenhäusern. Der BQS-Qualitätsreport zeigt, dass die deutschen Krankenhäuser eine Versorgung auf hohem Qualitätsniveau gewährleisten.

\section{Ungebrochener Trend zur Verbes- serung der Krankenhausergebnisse „Der Trend zur Verbesserung der Kran- kenhausergebnisse ging 2007 ungebro- chen mit hoher Dynamik weiter. Die Verbesserungsmaßnahmen greifen also.}

Trotzdem fallen immer wieder einzelne Krankenhäuser auf, die das geforderte Qualitätsniveau nicht erreichen“, sagte Dr. Christof Veit, Geschäftsführer der BQS. „Bei 20 der 194 Qualitätsindikatoren erreichen wir im Gesamtergebnis unser Ziel nicht, oder es sind ungewöhnlich viele Krankenhäuser auffällig. Dieser Verbesserungsbedarf wird analysiert, und es werden möglichst effektive Lösungen gesucht“, erläuterte Veit.

\section{Qualitätsberichte zur Analyse des Verbesserungsbedarfs}

Der Gesetzgeber hat mit dem GKV-WSG 2007 in $\S 137$ a SGB V dem Gemeinsamen Bundesausschuss (G-BA) auferlegt, eine Institution für Qualitätssicherung mit dieser Aufgabe zu beauftragen. Auf der Grundlage einer europaweiten Ausschreibung wurde anstelle der BQS das AQUA-Institut für angewandte Qualitätsförderung und Forschung im Gesundheitswesen, Göttingen, mit dieser Aufgabe betraut. Es wird ab 2010 die Qualitätsberichte veröffentlichen.

Die Aktivitäten des G-BA in der Qualitätssicherung sind durch dieses Ausschreibungsverfahren nicht beeinträchtigt worden, stellt der G-BA-Vorsitzende Dr. Rainer Hess fest. Dies zeigten die gefassten Beschlüsse zur Neustrukturierung der Qualitätsberichte der Krankenhäuser und die damit verbundene systematisierte Berücksichtigung der Ergebnisse des strukturierten Dialoges in der Berichterstattung. Als Meilenstein wertet Hess die jüngsten Beschlüsse zur Transparenz der Ergebnisse der Behandlung von Früh- und Neugeborenen in darauf spezialisierten Krankenhausabteilungen.

\section{Suchdienst für rasche Auskunft} und Vergleichsmöglichkeit

Wer ein geeignetes Krankenhaus sucht, muss nicht alle Qualitätsberichte der
Häuser einzeln abrufen. Er kann sich auch der Suchdienste der Krankenkassen bedienen, die eine mehr oder weniger einfache Auskunft samt Vergleichsmöglichkeit offerieren.

Der Klinik-Lotse der Ersatzkassen (www.klinik-lotse.de) stellt alle wesentlichen Informationen aus den Qualitätsberichten in übersichtlicher Form dar. Komfortable Suchfunktionen ermöglichen Vergleiche zwischen den verschiedenen Einrichtungen. Ebenso nutzerfreundlich ist der BKK Klinikfinder (www.bkk-klinikfinder.de). Der AOK Krankenhaus-Navigator dagegen ist exklusiv nur für registrierte AOK-Versicherte zugänglich. An einweisende Ärzte richtet sich das AOK-Klinik-Konsil, in dem die Qualitätsdaten aus dem Jahr 2006 zusammengefasst sind. Das KlinikKonsil bietet einen Recherchepfad zu den Versorgungsschwerpunkten an.

Klaus Schmidt, Planegg

\section{Wo ist die meiste Erfahrung?}

Patienten können sich mithilfe der Qualitätsberichte über alle sie interessierenden Fragestellungen rund um die Krankenhausversorgung informieren: Welche Klinik hat die meiste Erfahrung? Wo finde ich die Klinik, die sich am meisten für die Fort- und Weiterbildung ihrer Mitarbeiter engagiert? Wo gibt es welche Spezialisten für bestimmte Operationen?

Zum Beispiel enthalten die Qualitätsberichte die 30 häufigsten Diagnosen, die in den einzelnen medizinischen Fachbereichen der Klinik gestellt worden sind, ferner die 30 häufigsten Operationen, die von der Klinik durchgeführt wurden. Kliniken, die komplexe Operationsleistungen mit Mindestmengenvorgaben anbieten, müssen neben der Anzahl der durchgeführten Operationen auch Angaben über aufgetretene Komplikationen im Qualitätsbericht dokumentieren. Auf diese Weise erhalten die Patienten einen Überblick, wie viel Erfahrung die Ärzte eines Krankenhauses im Umgang mit bestimmten operativen Eingriffen haben. 